Enfermagem Brasil 2018;17(4):346-53

\title{
ARTIGO ORIGINAL \\ Prevalência de diabetes mellitus em idosos da zona rural no Sul do Brasil
}

Letícia Pilotto Casagranda*, Celmira Lange, D.Sc. ${ }^{* *}$, Juliana Graciela Vestena Zillmer, D.Sc. ${ }^{* *}$, Fernanda dos Santos ${ }^{\star * * *}$, Denise Somavila Przylynski Castro ${ }^{\star * * *}$, Patrícia Mirapalheta Pereira de Llano, D.Sc. ${ }^{* * * *}$

*Enfermeira, Doutoranda em Ciências da Saúde pelo Programa de Pós Graduação da Faculdade de Enfermagem UFPel, Docente no Centro Universitário Cenecista de Osório, **Enfermeira, Professora Associada da Faculdade de Enfermagem UFPel, Coordenadora da Pesquisa Pelotas/RS, ${ }^{* *}$ Enfermeira, Professora Adjunta da Faculdade de Enfermagem UFPel, ${ }^{* * * * E n f e r m e i r a, ~ D o u t o r a n d a ~ e m ~ C i e ̂ n c i a s ~ d a ~ S a u ́ d e ~ p e l o ~ P r o g r a m a ~ d e ~ P o ́ s-G r a d u a c ̧ a ̃ o ~ d a ~}$ Faculdade de Enfermagem da UFPel, Enfermeira na Prefeitura Municipal de Lajeado,

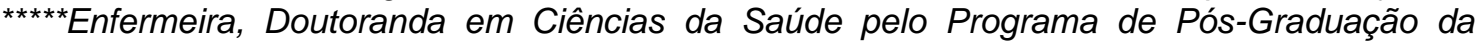
Faculdade de Enfermagem UFPel, Enfermeira no Hospital Universitário São Francisco de Paula, ${ }^{* * * * *}$ Enfermeira, Membro do Núcleo de Condições Crônicas e Suas Interfaces (NUCCRIN), Pelotas/RS

Recebido em 26 de maio de 2017; aceito em 4 de abril de 2018.

Endereço de correspondência: Letícia Pilotto Casagranda, Rua Gomes Carneiro 01/202, Pelotas RS, E-mail: cissapc@yahoo.com.br; Celmira Lange: celmira_lange@terra.com.br; Juliana Graciela Vestena Zillmer: juzillmer@gmail.com; Fernanda dos Santos: drenffernanda@gmail.com; Denise Somavila Przylynski Castro: deprizi@gmail.com; Patrícia Mirapalheta Pereira de Llano: pati_llano@yahoo.com.br

\section{Resumo}

Objetivo: Avaliar a prevalência de diabetes mellitus autorreferido em idosos residentes na zona rural. Métodos: Pesquisa quantitativa de caráter descritivo e delineamento transversal, com idosos de dez Unidades Básicas de Saúde com Estratégia de Saúde de Família, de julho a outubro de 2014. Resultados: Dos 820 idosos entrevistados, 139 referiram ter Diabetes Mellitus (DM), sendo 131 do tipo II. A prevalência de DM foi de 16,9\%, 53,2\% dos idosos estavam na faixa etária de 60 a 69 anos, 65,6\% eram do sexo feminino, 87,8\% de cor branca, 79,6\% tinham renda de até dois salários mínimos e 43,9\% tinham o ensino fundamental incompleto. Destaca-se o alto índice de peso em excesso, entre as complicações a retinopatia, a neuropatia e a nefropatia. A maioria dos idosos relatou sua autopercepção de saúde como boa ou ótima. Conclusão: Encontrou-se alta prevalência de DM, acima do índice mundial, bem como alto índice de complicações. Esta doença é desafiadora para os serviços de saúde. Ressalta-se a importância de profissionais de saúde manter conhecimentos acerca da doença, etiologia e sintomas, para que se possa intervir nos cuidados a esta patologia, bem como na prevenção de complicações.

Palavras-chave: Enfermagem, idoso, zona rural, diabetes mellitus.

Abstract

Prevalence of diabetes mellitus in elderly of the countryside in southern Brazil

Objective: Evaluating the prevalence of diabetes mellitus among elder people living in the rural area. Methods: Descriptive and cross-sectional characteristics, with elderly people from ten Healthcare Units with Family Health Strategy, from July to October of 2014. Results: Out of 820 elders interviewed, 139 reported having Diabetes Mellitus (DM), being 131 of type two resulting in a prevalence of DM 16,9\%. Other characteristics related were: $53,2 \%$ of the elders were from 60 to 69 years old, $65,6 \%$ were women, $87,8 \%$ had white complexion, $79,6 \%$ had an income of at most two minimum wages and $43,9 \%$ did not complete elementary school. The fact that most of them presented overweight can be highlighted, and among the common complications, retinopathy, neuropathy, and nephropathy were prevalent. Most of the interviewed reported a self-referred health as good or great. Conclusion: High prevalence of DM was observed, above the world average, as well as a high number of complications. This disease presents itself as challenging for healthcare services. It is important to emphasize the importance of health 
professionals to maintain knowledge about the disease, etiology and symptoms, in order to intervene in the care of this pathology, as well as in the prevention of complications.

Key-words: Nursing, aged, rural areas, diabetes mellitus.

\section{Resumen}

Prevalencia de diabetes mellitus en adultos mayores de la zona rural en el Sur de Brasil

Objetivo: Evaluar la prevalencia de diabetes mellitus auto-referido en adultos mayores residentes en el medio rural. Métodos: Investigación cuantitativa de carácter descriptivo y delineamiento transversal, con mayores de diez Unidades Básicas de Salud con Estrategia de Salud de la Familia, periodo de julio a octubre de 2014. Resultados: De los 820 adultos mayores entrevistados, 139 mencionaron tener diabetes mellitus (DM), siendo 131 del tipo II. La prevalencia de DM fue $16,9 \%, 53,2 \%$ de las personas de edad tenían entre 60 y 69 años, $65,6 \%$ del sexo femenino, $87,8 \%$ blancos, $79,6 \%$ con rendimientos hasta dos salarios mínimos y $43,9 \%$ con educación primaria incompleta. Se destaca el alto índice de peso en exceso, entre complicaciones retinopatía, neuropatía y la nefropatía. La mayoría de los adultos mayores relató su auto-percepción de salud como buena u óptima. Conclusión: Se encontró alta prevalencia de DM, superior al índice mundial, bien como alto índice de complicaciones. Esta enfermedad es desafiadora para los servicios de salud. Se resalta la importancia de los profesionales mantener conocimiento acerca de la enfermedad, etiología y síntomas, para que se pueda intervenir en los cuidados a esta patología, bien como la prevención de complicaciones.

Palabras-clave: Enfermería, anciano, medio rural, diabetes mellitus.

O Diabetes Mellitus (DM) vem sendo considerado uma doença devastadora para a saúde pública. Estima-se que no mundo 425 milhões de pessoas adultas são diabéticas, e a cada oito segundos uma pessoa vem a óbito devido a esta patologia. No Brasil, cerca de 13 milhões de pessoas possuem DM. Já no Rio Grande do Sul, estima-se que 135.075 mil habitantes são diabéticos, e no município do estudo há 4.978 mil pessoas com DM. Destaca-se ainda que para o ano de 2040, 642 milhões de pessoas, a nível mundial, provavelmente serão diagnosticadas com DM [1-3].

Esses dados em nível mundial nos levam a um grande cenário dramático no que se refere ao aumento desta condição crônica de saúde, tornando-se necessária a realização de prevenção e promoção desta patologia. A DM quando acometida, porém sem realizar 0 tratamento adequado, é uma das principais causas de doenças cardiovasculares, cegueira e insuficiência renal [4].

Além disso, o DM, dentre todas as doenças crônicas, destaca-se pela sua alta mortalidade entre os idosos. Por possuir uma evolução muitas vezes insidiosa e em muitos casos trazer consequências que podem ser agravantes, é temido por esta população, prejudicando sua qualidade de vida [5]. Contudo, observa-se na literatura que pesquisas com a temática idoso vêm ocorrendo principalmente em zonas urbanas e, desta forma, gera-se uma lacuna no que se refere ao contexto idoso rural [6].

E quando se fala em contexto rural, é preciso ter um olhar diferente sobre esta população. O meio rural apresenta um estilo de vida diferente de quem vive em áreas urbanas, e suas principais atividades econômicas estão voltadas para a produção de matéria prima, como a agrícola e a pecuária. Os idosos enfrentam algumas dificuldades, como o difícil acesso, dificuldades na linguagem - nestas localidades utiliza-se muito o dialeto local - e a condição climática, visto que as estradas em sua maioria são de chão batido e, quando chove em grande quantidade, tem-se muito barro, dificultando a passagem de veículos [7]. Portanto, é relevante um estudo com idosos que vivem neste contexto.

Diante do exposto, este estudo visa contribuir com a temática do idoso no contexto rural e com o diagnóstico de DM. Assim, o objetivo do estudo é avaliar a prevalência de diabetes mellitus autorreferido em idosos residentes na zona rural.

Este estudo está vinculado a um projeto de uma pesquisa quantitativa de caráter descritivo e delineamento transversal, sendo portanto um subprojeto. Foi desenvolvido entre a 
população idosa com 60 anos ou mais, de ambos os sexos, residentes na zona rural do munícipio de Pelotas/RS e que frequentavam Unidades Básicas de Saúde com Estratégia Saúde da Família (UBS/ESF). A Zona rural do munícipio conta com 12 UBS, porém fizeram parte desta pesquisa dez, pois estas possuem ESF.

Por tratar-se de um trabalho descritivo, este não exige cálculo de tamanho/poder amostral, uma vez que não pretende realizar testes de associação. Desta forma, utilizou-se a população obtida no cálculo amostral da pesquisa "Prevalência e fatores associados à síndrome da fragilidade na população idosa", da qual este trabalho deriva. A população idosa da zona rural de Pelotas é composta por 3.395 habitantes [2], havendo 2.920 idosos cadastrados nas ESF. Para o estudo principal foi determinada uma amostra de 834 idosos, já consideradas perdas e recusas.

Foram excluídos do estudo os idosos sem condições de responder ao questionário e sem acompanhante que convivesse ou conhecesse a história do idoso no momento da entrevista, os que estivessem viajando no momento da coleta daquela localidade, privados de liberdade por decisão judicial, ou que estivessem residindo em Instituições de Longa Permanência. Do total de 834 idosos contemplados a participar da pesquisa, nove foram considerados como perda e cinco como recusas. Assim, foram entrevistados 820 idosos.

As entrevistas foram realizadas no domicílio ou nas UBS, de acordo com a preferência de cada idoso, por entrevistadores devidamente capacitados. Contou-se com a colaboração dos agentes comunitários de saúde para a localização das residências dos idosos sorteados. A coleta de dados ocorreu nos meses de julho a outubro de 2014.

As informações foram obtidas por meio de questionário composto por questões fechadas, organizadas em blocos. A variável dependente deste estudo foi o DM autorreferido e as variáveis independentes foram características sociodemográficas e econômicas relacionadas à patologia de DM, aos hábitos comportamentais, estilo de vida, atividades desenvolvidas pelo idoso e autopercepção da saúde.

A pesquisa seguiu os princípios da Resolução do Conselho Nacional da Saúde 466/2012 [2]. O projeto foi cadastrado na Plataforma Brasil e aprovado pelo Comitê de Ética, do qual recebeu o parecer favorável de número 649.802. Aos idosos que concordaram em participar do estudo foi entregue e assinado o Termo de Consentimento Livre e Esclarecido (TCLE).

Os dados coletados foram digitados no programa Epi Info® 6.04 com dupla entrada e checagem de consistência. Para a análise do desfecho, realizou-se a estatística descritiva das variáveis pertinentes empregando-as o programa computacional Stata® 11.1.

Resultados

A prevalência de diabetes autorreferido na população idosa da zona rural foi de $16,9 \%$. A maioria foi representada pelo sexo feminino, faixa etária de 60 a 69 anos, da cor branca, residindo com companheiro e possuindo escolaridade de um a três anos. O consumo de tabaco e álcool foram referidos por menos da metade dos idosos diabéticos. Quanto à realização de atividade laboral, a maioria referiu que já realizou estas atividades. Estes dados podem ser observados na Tabela I.

Quanto ao tipo de DM, houve predominância de Diabetes Mellitus Tipo 2 (DM2) $(92,3 \%)$. Apenas 3 idosos referiram ter DM1 $(2,1 \%)$ e cinco não souberam informar qual o tipo de DM que possuíam. O motivo pela maior presença de DM2 pode ser explicado pelo estudo ser desenvolvido com idosos, e este tipo de diabetes acomete principalmente adultos e idosos com histórico familiar deste tipo de patologia e excesso de peso. 
Tabela I - Características dos idosos residentes na zona rural com diabetes mellitus autorreferido, segundo variáveis sociodemográficos, socioeconômicos e hábitos comportamentais $(N=139)$. Pelotas/2014.

\begin{tabular}{l|l|l}
\hline Variável & $\%$ & $\mathbf{n}$ \\
\hline Sexo & 65,6 & \\
Feminino & 34,4 & 41 \\
Masculino & & 48 \\
\hline Faixa etária & 53,2 & 74 \\
60-69 anos & 33,1 & 46 \\
70-79 anos & 13,7 & 19 \\
Acima de 80 anos & & \\
\hline Cor da pele & 87,8 & 122 \\
Branca & 12,2 & 17 \\
Não branca & & \\
\hline Situação conjugal & 71,9 & 100 \\
Com companheiro & 28,1 & 39 \\
Sem companheiro & & \\
\hline Escolaridade* & 13,7 & 19 \\
Analfabetos & 43,9 & 61 \\
1 a 3 anos & 36,7 & 51 \\
4 a 7 anos & 5,0 & 7 \\
8 anos ou mais & & \\
\hline Renda & 79,6 & 111 \\
Até 2 salários mínimos & 20,4 & 28 \\
\hline ou mais salário mínimo & 5,8 & 8 \\
\hline Tabagismo & 25,2 & 35 \\
\hline Etilista & & \\
\hline Atividade laboral & 29,5 & 45 \\
Sim & 66,2 & 92 \\
Não, mas já realizou & 4,3 & 6 \\
Nunca & &
\end{tabular}

*Um idoso não soube informar a escolaridade.

Tabela II - Cuidados realizados, autopercepção de saúde e Índice de Massa Corporal dos idosos com diagnóstico de Diabetes mellitus autorreferido (N=139). Pelotas/2014.

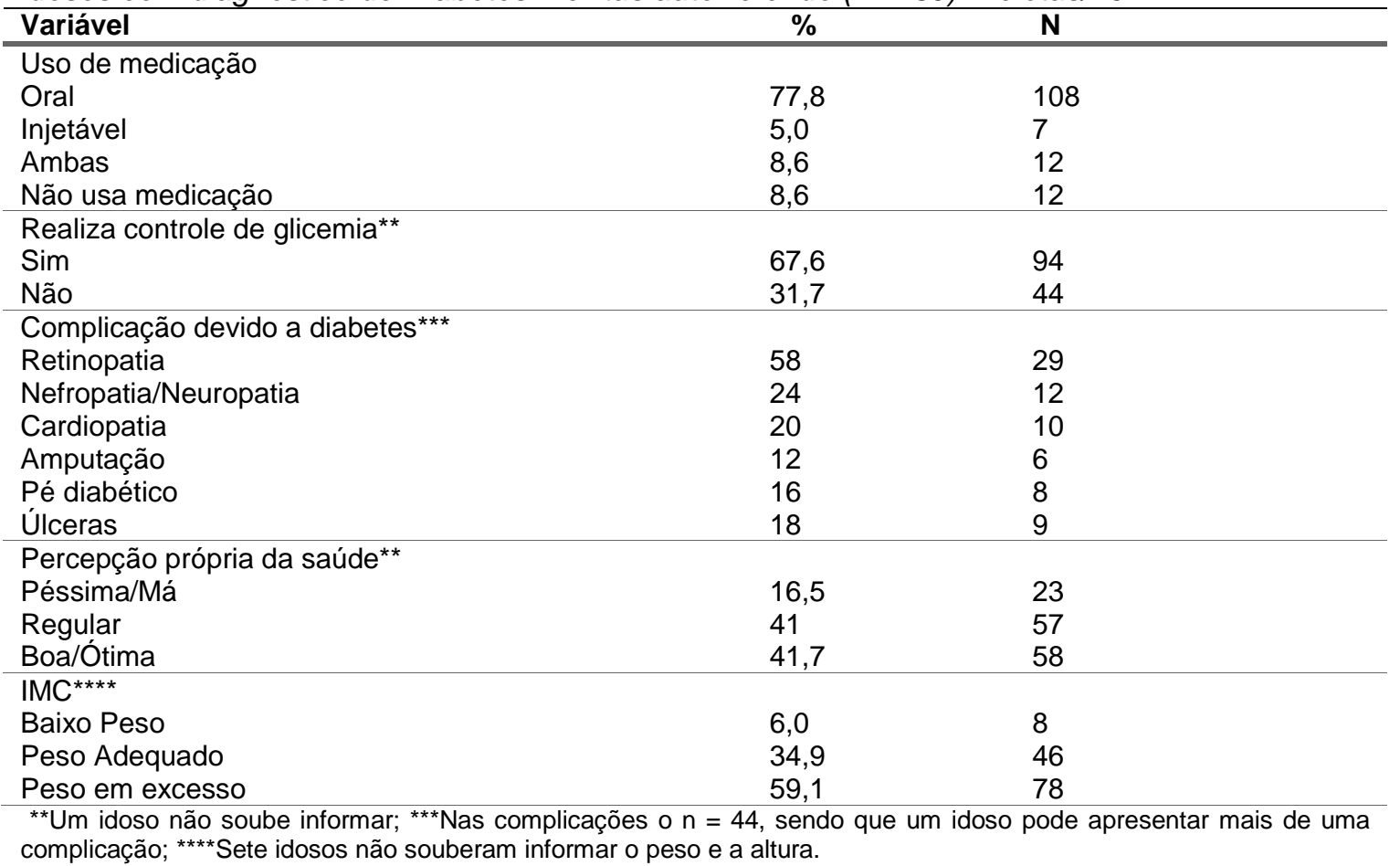


O uso de medicamentos foi citado pela maioria dos idosos, sendo a medicação oral a mais utilizada. Quando questionados sobre a realização do controle de glicemia, a maioria dos idosos, afirmou manter o controle.

As complicações, devido ao diagnóstico de DM, acometeram 44 idosos e estas questões, por serem de múltiplas escolhas, considerou-se a probabilidade de um idoso poder apresentar mais de uma complicação.

Destaca-se ainda, a autopercepção da saúde, em que a maioria dos idosos a classifica como boa/ótima. Outro dado que chamou a atenção foi quanto ao IMC, em que a maioria encontra-se em excesso de peso.

Discussão

A prevalência de DM neste estudo foi de $16,9 \%$, sendo considerada superior à prevalência de diabetes mellitus na população mundial que é de $9 \%$ e inferior a regiões como a ilha do pacífico que é de 33\% [3].

Em relação às características demográficas, houve predominância do sexo feminino $(65,6 \%)$. A maior prevalência deste sexo já foi observado em outros estudos, e também pode estar ligado ao fato de as mulheres serem mais conscientes quanto aos cuidados à sua saúde e por procurarem mais os serviços de saúde. Porém, na literatura, há uma discordância desta prevalência no sexo feminino, quando são utilizadas medidas bioquímicas, a prevalência de DM autorreferida foi de $42,6 \%$ no sexo masculino [9].

Quanto à faixa etária, observou-se uma maior predominância em idosos, cuja idade varia de 60 a 69 anos (53,2\%). Estes dados assemelham-se ao estudo cujo objetivo foi identificar a prevalência de diabetes mellitus referidos e seus fatores associados em idosos residentes no munícipio de Campinas, Paraíba, em que $80 \%$ encontram-se na mesma faixa etária [11]. Ainda considerando a idade dos idosos, segundo dados do Vigitel [12], a doença é mais comum com o avanço da idade, especialmente após os 45 anos, e um quarto dos indivíduos (24,4\%) com 65 anos ou mais referem o diagnóstico de DM. Dados estes semelhantes aos Estados Unidos (EUA), em que 29,1 milhões de pessoas são diabéticos, desses 13,4 milhões estão na faixa etária de 45 a 64 anos, e 11,2 milhões acima de 65 anos [13].

Neste estudo houve predominância da pele cor branca, conforme observado na Tabela I. Na literatura, encontrou-se divergências entre a prevalência de DM em brancos e não brancos. Alguns estudos referem que o DM é mais constante em afrodescendentes, pois eles são mais vulneráveis a hiperinsulinemia e a resistência à insulina, causando principalmente o DM2. Essa prevalência em afrodescendentes se dá pelo fato que no Brasil há uma grande mistura racial dos cruzamentos de índios nativos, colonizadores e dos negros africanos oriundos do oeste da África. Já em outros estudos, mostra-se a frequência maior em pessoas cuja pele é branca. Porém, outra pesquisa refere-se que os fatores ambientais e comportamentais também sejam considerados, como o exemplo de maior prevalência de diabéticos japoneses residentes nos EUA do que no Japão [14].

Neste estudo, somente $5 \%$ dos idosos estudaram oito anos ou mais, a maioria estudou de um a três anos. De acordo com os dados do Vigitel [12], quanto maior o grau de escolaridade, menor é a frequência de DM. A baixa escolaridade também pode acarretar problemas aos diabéticos que necessitam de um plano de cuidado. Observa-se no estudo realizado no Centro de Diabetes Alemão, a evidência de que quanto mais baixa a escolaridade, maior a interferência na aprendizagem sobre o autocuidado, prejudicando desta forma a prevenção das complicações, diferente dos que possuem maiores formações, pois estes têm um maior acesso aos serviços de saúde $[15,15]$. Outro fator que pode explicar essa baixa escolaridade na zona rural é o período de estudos ser o mesmo em que começam a contribuir com o trabalho no campo [17].

Ressalta-se que nesta época, a escolaridade em localidades rurais eram disponibilizadas somente nas séries iniciais, devido à má distribuição de escolas e também pelo fato de muitas vezes não haver professor na localidade, e posteriormente para dar continuidade aos estudos era necessário deslocar-se até a área urbana mais próxima. Contudo, nem todas as zonas rurais possuíam algum tipo de transporte que pudesse deslocar os alunos até a escola e, desta forma, as pessoas acabavam se direcionando ao trabalho no campo [18].

Diante do exposto, ressalta-se a permanência dos idosos na zona rural podendo estar atrelada ao início precoce nas atividades ligada ao campo. Estudo relata que as enxadas utilizadas para carpir eram produzidas de acordo com a estatura da criança, para que 
pudessem contribuir. Nesse estudo, ainda identificou-se que $91 \%$ dos entrevistados desempenham funções voltadas ao campo, $30,4 \%$ na ordenha de vaca e $73,9 \%$ cuidando de animais [17].

Quanto à renda, a maioria recebe até dois salários mínimos. Estudos evidenciam que quanto menor for a renda mensal de idosos diagnosticados com DM, maiores são as chances de virem a óbito. A renda pode ser um fator desencadeante para a não adesão de tratamentos destes pacientes diabéticos. Por esse motivo, é de extrema importância considerar as necessidades básicas dos indivíduos, para que desta forma se possa designar qual o melhor tratamento a ser seguido $[19,20]$. Além destas importâncias, ressalta-se que o profissional de saúde necessita utilizar um linguajar que favoreça o entendimento destes idosos, quantos aos cuidados que necessitam ser realizados para controle da patologia.

A alta prevalência de DM2 neste estudo pode estar associada ao fato da pesquisa ser com a população idosa, e este tipo de patologia é mais propenso a ser desenvolvido por esta faixa etária. Esta maior prevalência também pode ser um fator para explicar o maior número do uso de hipoglicemiantes orais. Geralmente, a DM2 pode evoluir durante anos antes que seja necessário o uso de insulina para maior controle [3]. Em relação às complicações, neste estudo a retinopatia foi a que mais acometeu. Estes dados assemelham-se ao estudo, cujo objetivo foi analisar a prevalência de diabetes em idosos e as medidas de controle adotadas [21].

A autopercepção de saúde destes idosos, apesar da patologia clínica, foi considerada como boa ou ótima, concordando com estudo em que foi avaliada a percepção de saúde de idosos residentes em um munícipio do interior do Rio Grande do Sul, em que dos 274 entrevistados, $13,5 \%$ são diabéticos, e classificaram sua autopercepção de saúde como boa [22].

Em um estudo realizado com idosos rurais e urbanos, evidenciou-se que os idosos que residem na zona rural desenvolvem mais a patologia de $\mathrm{DM}$, enquanto aqueles que vivem na zona urbana são mais propícios a desenvolverem doenças relacionadas ao sistema nervoso central. Esta expansão de DM pode estar associada ao aumento de prevalência de obesidade na população mais velha. Há evidências que no meio rural existem maiores taxas de sobrepeso e obesidade, e esta é considerada um fator de risco para o DM. Contudo, os dados revelados pelo Vigitel [13] afirmam que esta taxa de obesidade começou a declinar após os 65 anos [20,23-24].

Quanto ao tabagismo e etilismo, não houve valores significativos neste estudo, conforme se observa na tabela I. Estes dados são semelhantes ao estudo em que dentre os idosos que referiram fazer uso de bebida alcoólica, a maioria $(57,1 \%)$ referiu beber moderadamente em algumas ocasiões [21].

Diante dos fatos expostos, identificar a presença desta patologia precocemente, é uma forma de amenizar os problemas decorrentes da DM. Desta forma, a pessoa com esta doença necessita realizar o tratamento ao qual está submetido, de forma correta, proporcionando uma melhora em sua qualidade de vida.

Conclusão

Uma das limitações deste estudo é pelas informações coletadas terem sido autorreferidas. Ao final do estudo, prevaleceu o sexo feminino, a cor branca, a baixa escolaridade e o alto índice de sobrepeso e obesidade. A prevalência de DM foi de 16,9\%, estando acima da média mundial que é de 10\%. A predominância da cor branca pode estar atrelada ao fato de que a localidade estudada é descendente de alemães.

O DM é considerado uma doença desafiadora para os serviços de saúde. A cada ano que passa atinge mais a população, tornando-se um agravante na saúde pública. Desta forma, é de extrema importância que todos os profissionais da área da saúde estejam atualizados sobre os riscos que o DM implica nos idosos, para que seja possível realizar o acompanhamento necessário, repassando as informações e cuidados, principalmente entre os idosos, por muitos desconhecer a sua patologia.

Destaca-se, ainda, a realização da pesquisa ser em localidade rural, em que há uma lacuna de pesquisas realizadas e, desta forma, este estudo contribuiu para que outros pesquisadores possam realizar estudos voltados para esta temática. 
1. International Diabetes Federation (IDF). Diabetes Atlas de la fid. 2015- Atualização. 8 ${ }^{\underline{a}}$ ed; 2017.

2. Instituto Brasileiro de Geografia e Estatística (IBGE). Distribuição da população por sexo, segundo os grupos de idade Pelotas (RS)-2010. [citado 2018 Abr 3]. Disponível em:

http://www.censo2010.ibge.gov.br/sinopse/webservice/frm_piramide.php?codigo=4314 $40 \&$ corhomem $=3 \mathrm{~d} 4590$ \& cormulher $=9 \mathrm{cdbfc}$

3. Sociedade Brasileira de Diabetes (SBD). Número do Diabetes no Brasil.[online] 2018. [ citado 2018 Abr 3]. Disponível em: http://www.endocrino.org.br/numeros-do-diabetesno-brasil/

4. World Health Organization (WHO). World health statistics 2012. [citado 2018 Abr 3]. Disponível em: http://www.diabetes.org.br/ultimas/o-avanco-do-diabetes-no-mundosegundo-a-oms

5. Matias COF, Matias COF, Alencar BR. Qualidade de vida em idosos portadores de diabetes mellitus tipo 2 atendidos em Unidades Básicas de Saúde de Montes Claros/MG. Revista Brasileira de Qualidade de Vida 2016;8(2):119-29.

6. Tavares DMS, Santos TG, Dias FA, Bolina AF, Ferreira PCS. Health and leisure among elderly rural diabetics with and without indications of depression. Rev Enferm UERJ 2015;23(4):548-55.

7. Llano PMP, Lange C, Casagranda LP, Santos F, Castro DSP, Pinto AH et al. Research experience developed with elderly who live in the countryside. J Nurs Health 2015;5(2):153-61.

8. Brasil. Ministério da Saúde. Conselho Nacional de Saúde. Resolução 466, de 12 de dezembro de 2012: diretrizes e normas regulamentadoras de pesquisa envolvendo seres humanos. Brasília: Ministério da Saúde; 2012.

9. Iser BPM, Stopa SR, Chueiri OS, Szwarcwald CL, Malta DC, Monteiro HOC et al. Selfreported diabetes prevalence in Brazil: results from National Health Survey 2013. Epidemiol Serv Saúde 2015;24(2):305-14.

10. Schimidt MI, Hoffmann JF, Diniz MFS, Lotufo PA, Griep RH, Bensenor IM et al. High prevalence of diabetes and intermediate hyperglycemia - The Brazilian Longitudinal Study of Adult Health (ELSA-Brasil). Diabetology \& Metabolic Syndrome 2014;6:123.

11. Menezes TN, Sousa NDS, Moreira AS, Pedraza DF. Self-reported diabetes mellitus and associated Rev Bras Geriatr Gerontol 2014;17(4):829-39.

12. Brasil. Ministério da saúde. Secretária de Vigilância em Saúde. Departamento de Vigilância de doenças e agravos não transmissíveis e promoção da saúde. Vigitel Brasil 2014: vigilância de fatores de risco e proteção para doenças crônicas por inquérito telefônico / Ministério da Saúde, Secretaria de Vigilância em Saúde, Departamento de Vigilância de Doenças e Agravos não Transmissíveis e Promoção da Saúde. Brasília: Ministério da Saúde; 2015.

13. National Center for Chronic Disease Prevention and Health Promotion. National Diabetes statistics reports, 2014. [online]. [citado 2018 Abr 3]. Disponível em: http://www.cdc.gov/diabetes/pubs/statsreport14/national-diabetes-report-web.pdf

14. Brito IC, Lopes AA, Araújo LMB. Associação da cor da pele com diabetes mellitus tipo 2 e intolerância à glicose em mulheres obesas de Salvador, Bahia. Arq Bras Endocrinol Metab 2001;45(5):475-80.

15. Cortez DN, Reis IA, Souza DAS, Macedo MML, Torres HC. Complications and the time of diagnosis of diabetes mellitus in primary care. Acta Paul Enferm 2015;28(3):250-5.

16. Genz J, Haastert B, Müller H, Verheyen F, Cole D, Rathmann W et al. Socioeconomic factors and effect of evidence-based patient information about primary prevention of type 2 diabetes mellitus - are there interactions? BMC Res Notes 2014;7(1):541.

17. Rempel $C$, Haetinger $C$, Sehnem $E$. Reflexões de idosos sobre as relações entre 0 trabalho rural, problemas de coluna e postura corporal. Estud Soc Agric 2013;21(2):289-307.

18. Maia EM. Educação rural no Brasil: O que mudou em 60 anos? Revista da Associação Nacional de Educação 1982;1(3):5-11.

19. Caldas, ACS, Morais, MMP, Nunes, FDO, Dias RS, Sousa SMA. Characteristic of diabetic patients served in the ambulatory of a university hospital. Rev Pesq Saúde. 2018;18(1):41-44. 
20. Jannuzzi FF, Cintra FA, Rodrigues RCM, São-João TM, Gallani MCBJ. Adesão medicamentosa e qualidade de vida em idosos com retinopatia diabética. Rev Latinoam Enferm 2014;22(5):902-10.

21. Stopa SR, César CLG, Segri NJ, Goldbaum M, Guimarães VMV, Alves MCGP. Selfreported diabetes in older people: comparison of prevalences and control measures. Rev Saúde Pública 2014;48(4):554-62.

22. Borges AM, Santos G, Kummer JA, Fior L, Molin VD, Wibelinger LM. Self- perceived health in elderly living in a city in Rio Grande do Sul state. Rev Bras Geriatr Gerontol 2014;17(1):79-86.

23. Mendes LL, Gazzinelli A, Velásquez-Meléndez G. Factors associated with insulin resistance in rural populations. Arq Bras Endocrinol Metab 2009;53(3):333-9.

24. Silva EV, Paniz VMV, Laste G, Torres ILS. The prevalence of morbidity and symptoms among the elderly: a comparative study between rural and urban areas. Ciênc Saúde Coletiva 2013;18(4):1029-40. 